\title{
La radio universitaria en Iberoamérica: trascendencia y retos
}

\section{The college radio in Latin America: importance and challenges}

\author{
Marina Vázquez Guerrero \\ Profesora e Investigadora de la Licenciatura en Publicidad y Relaciones Públicas de la Escuela de Mercadotecnia \\ de la Universidad de Colima, México. \\ marina.vazquez.g@gmail.com
}

Resumen

Las primeras experiencias de radio universitaria que surgen en Iberoamérica se establecen en la Argentina. A lo largo del tiempo algunos países de la región han optado por abrir este tipo de emisoras que muestran un conglomerado sonoro e ideológico con diversos matices, en beneficio de su comunidad. Es un medio no lucrativo, que actualmente crece en toda la región, arropado por las ventajas que trae consigo la era digital e Internet. Radios con perfiles institucionales, comunitarios, estudiantiles se abren espacio para difundir desde la cultura y labor académica hasta la experimentación estudiantil. El presente trabajo es una investigación documental, que da cuenta de su parte histórica, así como de la trascendencia actual de los casos de México, Chile, Colombia, Argentina y Ecuador, haciendo un énfasis sobre los retos a los que se enfrenta este conglomerado que ha tardado en entrar a la nueva dinámica interactiva digital y en incorporar más producciones de contenido social, necesaria en estos tiempos donde los discursos comerciales abundan y la sociedad civil requiere de foros mediáticos confiables.

\begin{abstract}
The first experiences of college radio in LatinAmerica are established in Argentina. Over time some countries in the region have to opened this kind of stationss howing a diversity of sounds and ideological ideologies for the benefit of community. Is a non-profit media, which no wgrows throug houtthe regione mbraced by the advantages that come with the digital and Internet age. Institutional, community and university radios are opening spaces for supporting cultural and academic content to the student experimentation. The present study is a documentary research showing the historical part and the actual significance of the cases of Mexico, Chile, Colombia, Argentina and Ecuador, with an emphasis on the challenges facing this conglomerate that has been slow to enter the new digital interactive dynamics and productions incorporate more social content necessary in these times, where commercial speech abound and civil society requires reliable media fórums.
\end{abstract}

\footnotetext{
Palabras clave Radio universitaria, Iberoamérica, estudiantes, extensión universitaria.

Key College radio, Latin America,students, universityextension. words
}

○ Agradecimientos: Esta investigación contó con el apoyo de la Universidad de Colima, México. 


\section{Introducción}

En pleno siglo $\mathrm{XXI}$, inmersos como estamos en nuevas tecnologías simplificadas en dispositivos más complejos, la radio sigue presente, mutando y respondiendo a necesidades primarias de comunicación, entretenimiento, información local y educación, entre muchas otras.

Particularmente en América Latina se observa que no se pierde la magia de aquél artefacto que fuera una de las primeras herramientas de comunicación masiva, un tanto apoyados por la tradición y cultura de la escucha, pero también por el rezago económico y la brecha digital. Oír radio en una proporción grande de habitantes del continente, es la única fuente de acceso al mundo exterior o por lo menos, la más asequible, y de mayor credibilidad.

En este panorama, se encuentra inserta la Radio Universitaria, un medio de origen institucional que ofrece productos sonoros de interés general, con contenidos especializados y diversos. En términos generales la labor principal de toda emisora universitaria es la de difundir saberes hacia la sociedad y reflejar en sus contenidos los objetivos que como sistema educativo sigue, planteado de diferente manera según el país, de acuerdo a sus estatutos y reglamentos.

Al no ser un medio comercial - en la generalidad de casos-, su financiación proviene de la propia universidad, en la mayoría de las de América y Europa y de sus oyentes en Estados Unidos; por lo tanto, su organización interna, su personal y forma de trabajo no es homogénea y puede no seguir las pautas de una emisora convencional. No existen emisoras idénticas, ni patrones únicos. Se habla de por lo menos 14 modelos que parten de tres modos de producción radiofónica que van de lo institucional a lo comunitario y en pocos casos, lo comercial, y que se pueden dar como etapas en un mismo medio, de acuerdo a las políticas e historia de cada universidad.

No existen estudios a profundidad en la región que definan cuantitativamente su audiencia y solo algunos países han realizado investigaciones sobre sus contenidos y perfiles. Es un campo abierto, en el cual van explorando y aportando poco a poco unos cuantos colegas.

De las experiencias empíricas, podemos decir que este tipo de emisoras, que no deben su existencia a intereses empresariales, ofrecen en muchos casos otros discursos: son cercanas a colectivos, movimientos culturales, sociales y a nuevos formatos que no son 
producidos necesariamente por profesionales de la comunicación y el periodismo. Otra característica habitual, como sucede en México y España, es que la radio universitaria, no forma parte ni de los medios públicos ni de los comunitarios: "esto ha evitado que sigan políticas y patrones similares a los medios estatales, desarrollando una personalidad alterna e interesante, pero padecen la falta de beneficios económicos y legales, necesarios para su crecimiento y visibilidad social" (Vázquez, 2012, p.94).

En el siguiente artículo se busca mostrar un panorama descriptivo de las experiencias que actualmente se tienen en Iberoamérica ${ }^{1}$, en particular sobre los casos de Argentina, México, Chile, Colombia, España y Ecuador, que son los más documentados, así como la trascendencia y retos que existen y que tienen relación con los cambios tecnológicos, pero también con la necesidad de un cambio de mentalidad en aquellas radios poco flexibles y encerradas en una burbuja que no les permite crecer, integrar nuevos elementos ni aportar en los conflictos sociales.

La región latinoamericana, como se podrá ver, tiene un registro importante de radios universitarias y las experiencias han sido diversas y crecientes, al punto de que en los últimos años, países como España están dialogando y compartiendo prácticas y estrategias, generando un movimiento de intercambio y colaboración entre los dos continentes.

\section{Las primeras emisoras}

Desde hace algunos años, se ha venido afirmando y confirmando desde la región, que la primera radio universitaria en el mundo surgió casi a la par que las primeras experiencias sonoras, en Argentina, en abril de 1924, desde los micrófonos de la Universidad de La Plata (Rotman, 2014).

Sin embargo, existe documentación histórica en los Estados Unidos que asegura que la radio nació en su región y que contribuyeron "al desarrollo del medio y en la formación del sistema americano de radiodifusión comercial” (Vázquez, 2012) porque fueron los físicos e ingenieros quienes utilizaron estaciones de radio experimentales (Slotten, 2006) y también quienes emitieron los primeros programas. La Universidad de Wisconsin, en particular, a través de la señal de la WHA, transmitía de manera regular información meteorológica y económica (Faus, 1973). En esta Universidad se inició desde 1900 la experimentación

Entendiendo el término que abarca los países hispanohablantes de América Latina y Europa 
usando transmisores de chispa por profesores y estudiantes y en 1915 tuvieron la primera licencia experimental denominada 9XM, haciendo su primera transmisión de voz entre 1919 y 1920 (Miller, 2007). Su página oficial afirma que es la primera radio en América "Since 1917 stimulating, informative and entertaining companion" (WPR, 2014)2.

Slotten (2006) afirma que para 1925 - un año después del nacimiento de la radio universitaria argentina-, ya existían 124 emisoras universitarias en ese país norteamericano $y$, si bien hoy muchas no llevan el distintivo de "universitarias", fue porque en los años 70 , tras un movimiento de radios, se unieron todas las formas no lucrativas de radiodifusión a la National Public Radio (Martí, 1995). Actualmente en Estados Unidos, aquellas radios pioneras forman parte de la radio pública y reciben donativos de la audiencia pero también de ayuntamientos, universidades públicas y privadas; colegios, Estados federales y fundaciones de las importantes empresas del país como Ford, IBM, Xerox, etc. (Chaparro, 1998).

Las radios que mantienen esa figura de radios universitarias, hechas por miembros de su comunidad, son los college radio, que son realizados por estudiantes y operadas dentro del campus para emitir música alternativa (Araya, 2009). Las estaciones son de baja potencia y están ubicadas dentro de algún departamento de la universidad como parte de los programas de estudio que pueden ser: radio, TV, cine, periodismo, medios masivos, comunicación masiva, comunicación oral, etc. "De esta forma las estaciones operan bajo la dirección de alguna entidad académica" (Magaña, 2003) y por lo tanto la supervisión de las estaciones corre principalmente por parte de maestros y personal de staff, aunque hay alumnos que trabajan en la estación como managers para cubrir créditos requeridos para graduarse ${ }^{3}$.

Consensos históricos aparte, lo que buscamos resaltar en este apartado del artículo son las características de las emisoras pioneras en Iberoamérica y su contexto histórico, que ha sido diferente al norteamericano. Acá la radio inició con la misión de llevar la extensión cultural al pueblo, por medio de conferencias y otros formatos académicos.

En Latinoamérica, las universidades tuvieron acceso a la tecnología sonora, por el lugar relevante de estos centros educativos en el desarrollo del país, formando parte de los grupos de poder, como la iglesia. Las universidades que contaban con recursos en

2 Página web oficial de WPR [http://www.wpr.org/about-wpr / consulta: 13/12/2014].

3 Ibíd. pág. 5. 
los primeros años del desarrollo de la radio, vieron en ella una gran oportunidad para transmitir la cultura, difundir su filosofía, entre otras. Así lo manifiestan los documentos históricos de la pionera radio de la Universidad Nacional de la Plata, fundada en 1924 (ARUNA, 2014) y de la segunda que nació en 1931, en la Universidad Nacional del Litoral, debutando con pocas horas de emisión y una programación estrictamente cultural donde "se irradiaba música clásica, noticias del ámbito universitario, información agrícola ganadera, comentarios de arte y literatura, enseñanza de idiomas, divulgación científica, charlas y conferencias" ( LT10, 2015).

Le sigue en antigüedad la emisora cultural de la Universidad de Antioquia, en Colombia, que tuvo su señal en onda corta en 1933 pero que hasta 1938 tuvo permiso oficial ${ }^{4}$. En 1937 aparecen dos: en abril, la radio de la Universidad Técnica Federico Santa María en Valparaíso, Chile, y en junio, Radio UNAM, en México.

Por su parte, en España se tuvo un inicio muy diferente. La experiencia más antigua, aunque no con el modelo de radio que predomina, fue Radio UNED, productora de la Universidad Nacional de Educación a Distancia que tiene un acuerdo con Radio Nacional de España para emitir contenidos desde 1979 por Radio 3. Su objetivo es hacer una programación que contribuya a "llevar la educación superior a los núcleos de población, alejados de las grandes metrópolis, que no disponen de universidad" (UNED, 2015), labor que continúa realizando con el apoyo de las nuevas tecnologías de Internet, pero sin una emisora con señal constante. Su perfil aunque es de una emisora universitaria, es sobre todo de una radio educativa o formativa.

Por otra parte, antes de la llegada de Internet, las únicas emisoras universitarias que aparecieron fueron en ciudades donde era posible adquirir una frecuencia por vía legal o en forma de convenio, y para ello fue necesario contar con el apoyo de la institución, pero también con equipos entusiasmados por el proyecto con "el empeño de un reducido grupo de personas que pretendían tener un altavoz dirigido hacia la sociedad" (Fidalgo, 2009, p.04).

Por lo tanto, podemos decir que la emisora pionera se estableció en 1987 a iniciativa de estudiantes de la Universidad de la Laguna, en Tenerife. Esta radio, que se montó como un taller en los baños adaptados del colegio mayor San Fernando de la universidad, comenzó

4 Página oficial de la emisora cultural de la Universidad de Antioquiahttp://emisora.udea.edu.co/ (13/12/2014). 
llamándose Radio San Fernando y emitiendo "con un equipo muy rudimentario, casi prefabricado" (Capote, 2010) que sirvió para hacer emisiones experimentales e irregulares. Posteriormente, hacia 1992, en la celebración del bicentenario de la universidad "el vicerrector de extensión universitaria decide apostar decididamente por la radio y se reacondicionan unas instalaciones" (Capote, 2010) cambiando de nombre a Radio Campus. También se comenzó una etapa de enseñanza del medio más sistematizada, para que los estudiantes aprendieran realización, operación técnica, producción y guión, que posteriormente se fue ejerciendo con las nuevas generaciones.

En 1995 nace en Salamanca el segundo proyecto en el seno de la Facultad de Comunicación, con emisiones irregulares, pues se trataba de un taller de estudiantes. La radio se consolida hasta que pasó a ser gestionada por el gabinete de Comunicación y protocolo, con el uso de Internet, en 2001.

\section{Radios Universitarias en Latinoamérica}

En este continente es donde actualmente tiene presencia importante la radiodifusión universitaria. Aunque no existe un registro preciso, se calculan alrededor de 200 de habla hispana (Casajús, 2011; Vázquez, 2011). A través de las diferentes redes y asociaciones de radios, se pueden identificar algunas cifras que mostramos en la tabla 1, y que sin embargo no reflejan el número real, pues las agrupaciones tienen criterios limitantes para la totalidad de las experiencias sonoras. Por ejemplo, en México se registra la presencia de más de 85 frecuencias que administran 51 instituciones de Educación Superior, "sin contar por lo menos 24 productoras y radios online que amplían el grupo a más de 75 universidades haciendo radio" ( Vázquez, 2014) cuando la red oficial, menciona 38 instituciones con 70 frecuencias ( SINPRIES, 2015). Por su parte, Merayo (2007) registraba en Chile 30 y 10 más en Venezuela, cifras que no coinciden con lo reportado por la Red de Radios Universitarias de América Latina y el Caribe. 
Tabla 1. Radios universitarias afiliadas a redes por países

\begin{tabular}{|c|c|c|}
\hline PAÍS & NO. UNIVERSIDADES & NO. FRECUENCIAS \\
\hline Argentina (ARUNA) ${ }^{5}$ & 37 & 35 \\
\hline Chile (REUCH) $^{5}$ & 17 & 17 \\
\hline Colombia (RUC) $^{6}$ & 40 & 58 \\
\hline Ecuador (RRUE) $^{7}$ & 11 & 11 \\
\hline México (SINPRIES) & 38 & 70 \\
\hline TOTAL & 143 & 191 \\
\hline
\end{tabular}

Elaboración propia. Fuente: (RRULAC, 2015)

La suma confirmada por RRULAC es de 143 universidades que administran 191 frecuencias. Haciendo un recorrido por el continente, comenzamos mostrando las características de la radio primeriza.

\section{Argentina: Las primeras experiencias}

En Argentina existen más de 35 emisoras agrupadas en su mayoría en la Asociación de Radio Universitarias Nacional de Argentina (ARUNA). Aunque las primeras nacieron casi a la par de la radio comercial, se desarrollaron numéricamente después de 1983 "con el fin de la dictadura y la normalización de las casas de altos estudios" (Novelli y Hernando, 2011, p.15). Aquí hay varios modelos con objetivos diversos, como aquellas que buscan favorecer las prácticas estudiantiles o ser un foro de expresión de la comunidad universitaria, pasando por aquellas "que intentan convertir su medio de comunicación en un centro de investigación y experimentación y también aquellas, que dependiendo del rector, desarrollan una comunicación institucional muy marcada o de actos de gobierno" (Novelli y Hernando, 2011, p. 19). Como en otras experiencias, algunas tienen poco reconocimiento social por la forma en que es operada desde la institución, que las puede dejar por largos periodos sin presupuesto, sin capacitación docente y con demasiadas políticas de extensión intervencionistas.

\footnotetext{
5 La Asociación de Radios Universitarias Nacionales Argentinas (ARUNA)http://www.aruna.org.ar/home/ consulta:12/01/2015)

6 Red de Radios Universitarias de Colombia[http://www.radiouniversitaria.org consulta: /02/01/2015].

7 Red de radios Universitarias de Ecuador. http://www.rrue.org/?page_id=185 consulta: 02/01/2015)
} 
Se afirma por ello que la mayoría son medios más institucionalizados en el sentido de que "el Rectorado, el Consejo Superior, y el área de Extensión intervienen con responsabilidades básicas en su funcionamiento y reciben asistencia y recursos humanos de las carreras afines a la comunicación que la universidad tenga en sus planes de estudio" (Dido, 2008, p.03).

A pesar de ello, las radios en este país también se caracterizan por ser más abiertas a la participación social y expresión política, algo que en otros países latinoamericanos no es posible.

Muchos de los proyectos actuales de radios universitarias se reconocen justamente desde ese eje dialógico con la sociedad, donde la existencia y la programación de estas emisoras constituye una apuesta a trabajar las problemáticas sociales ya construir una idea de saber y de conocimiento social al servicio de la comunidad y en pos de justicia social (Morales, 2008, p.103).

En cuanto a la tecnología, hasta el año 2011 solo el 74\% de las emisoras tenían presencia en internet, ya sea mediante la combinación de sitio web y emisión online, o bien con una de ambas opciones ( Milito y Casajús, 2011).

\section{México: Los numerosos casos}

En este país, aunque desde el inicio de la radiodifusión predominó el servicio privado o comercial, la radio universitaria ha tenido su espacio y el desarrollo se fue dando paulatinamente. Un año después de que iniciara transmisiones Radio UNAM, se funda Radio Universidad de San Luis Potosí, en 1938 y posteriormente le seguirían las de Veracruz (1944), Autónoma de Chihuahua (1957) y la de Guanajuato (1961). Para 1979 había 16 universidades con emisoras y aunque hubo un periodo de crisis política donde no se emitieron permisos, a partir de 1991 comienza otro aumento en el número de frecuencias otorgadas. En esa década 13 universidades reciben su permiso y la Universidad de Tamaulipas estrena seis frecuencias que enlazan por vía satélite las principales regiones de su gran territorio (Vázquez, 2014). En el año 2000, con el cambio de siglo y de partido en el gobierno (PAN) once instituciones más reciben sus permisos y un número importante aumenta sus frecuencias. "Con este incremento que significó casi un 52\% de las existentes, México se ha convertido en el país de habla hispana con más universidades que tienen estaciones de radio" (Vázquez, 2012). 
Estas primeras radios tuvieron un respaldo económico fuerte de la institución que las creó, por lo que es posible encontrar en ellas una plantilla importante de empleados. Radio UNAM es la mayor de ellas, con la emisión de contenidos por tres frecuencias (AM, FM y onda corta) y más de 200 empleados. Sin embargo, actualmente la mayoría de radiodifusoras y productoras operan con un reducido personal propio y la participación de la comunidad que oscila entre las 30 a 60 personas al año. La mayoría tiene entre mil y tres milwatts, que les permite cubrir solo parte de sus regiones". Tienen voz, pero no se les escucha totalmente en su territorio" (Vázquez, 2014).

De acuerdo al tipo de universidad, hay una primera clasificación general de la radio mexicana: a través de las universidades públicas autónomas, quienes son mayoría y promueven contenidos más institucionales y culturales; las radios de los institutos tecnológicos que usan sus medios para la práctica estudiantil de las áreas técnicas y las instituciones privadas que cuentan con emisoras, la mayoría por internet, con un formato similar a los college radio norteamericanos donde hay una alta participación estudiantil y la promoción de artistas alternativos.

Al tener un número importante de radios, este es uno de los países que seguramente presenta casi todos los modelos de radio e incluso fusiones, pues co-habitan desde la organizada en un sistema regional como Red radio UdeG, de Jalisco, hasta las comunitarias experimentales que dependen de la participación estudiantil y emiten solo por internet, como Frecuencia CEM, del Estado de México.

En cuanto a los contenidos de su programación, la mayoría emite contenidos culturales o académicos, dejando pocos espacios para la divulgación de la ciencia, o la expresión de colectivos sociales.

A pesar de los cambios en la ley que se han dado tanto en 2006, con la denominada Ley Televisa, como en 2014, con la Reforma Constitucional en materia de Telecomunicaciones, las radios universitarias continúan con su labor, enfrentando los nuevos retos y adaptándose a las exigencias que les siguen impidiendo, principalmente, obtener recursos propios o mejorar sus condiciones técnicas de transmisión. 


\section{Colombia: entre comercial y social con tono juvenil}

En el caso de Colombia, si bien la primera radio nace en 1933 en la Universidad de Antioquía, su desarrollo fue tardío pues durante muchos años solo existían dos: la pionera y la radio de la Universidad Pontifica Bolivariana. "En los años ochenta comienzan a generalizarse los medios radiofónicos universitarios en todo el país" (Novelli y Hernando, 2011, p. 15).

En el territorio colombiano, la mayoría de radios nacieron como resultado de proyectos de individuos, que luego fueron acogidos por las universidades (Gaviria, 2008). La figura de este tipo de medio, que legalmente pertenece a una categoría denominada medios de interés público, donde también están las municipales, militares y policiacas, no tienen licencia de publicidad si pertenecen a universidades públicas.

En el caso de las emisoras privadas que se calculan son el 18\%, tienen una concesión que les permite recibir recursos, pero no todas recurren a esta posibilidad. La mayoría de estas radios emite música clásica y se habla de una audiencia de 100 mil oyentes tan solo en la capital bogotana (Gaviria, 2008).

Su red principal (RRUC) afirma que son 40 las universidades con radios, emitiendo a través de 58 frecuencias. De este grupo, el 94\% funciona bajo la supervisión de los departamentos de periodismo o de comunicaciones de cada universidad y poseen personal profesional remunerado y practicantes del área de comunicación social. Sin embargo, quienes son mayoría en estas radios son estudiantes, voluntarios y personal por horas (Zambrano, 2012).

Respecto al tipo de contenidos que se emiten por estas radios y que en su mayoría son diseñados y presentados por jóvenes, sobresale la programación de música de arte y programas didácticos. En un $43 \%$ "trasmiten contenidos enfocados a la sociedad actual y 42\% tiene temáticas específica" (Zambrano, 2012).

\section{Chile: voces críticas}

En Chile, si bien se tiene una visión de que los medios de comunicación están en manos de unos cuantos, vinculados a interés políticos y económicos (Jiménez y Muñoz, 2008), es uno de los pocos países donde la radio universitaria tiene un perfil diverso, por tener la posibilidad de recibir auspicios, sin perder sus objetivos sociales. 
Después de haber vivido una etapa "marcada por las restricciones acerca de lo que podía transmitirse y que no, e incluso con destrucción de archivos sonoros de gran valor" (Martín Pena, 2013, p. 57) hay un saldo positivo, pues actualmente se tienen alrededor de 30 concesiones de radio (Merayo, 2007) que coordinan 26 casas de estudios (Dahma, 2014). Poco más de la mitad de estas, forman parte de la Red de Radioemisoras Universitarias de Chile (REUCH) que tiene más de 30 años de existencia y es patrocinada por el Consejo de Rectores de las Universidades Chilenas.

En el país donde nació la tercera radio universitaria, la mayoría tiene una vocación cultural.

Radio Universidad de Chile, por ejemplo, funciona como toda una empresa de medios y cuenta con una audiencia superior a los 120 mil. Además de recibir auspicios por fondos concursables como el Fondart, Fondo de Medios, del Libro y de la Música, el financiamiento proviene de ingresos propios a través de convenios con instituciones y publicidad de empresas reconocidas como Movistar, Banco del Estado o viñedos Emiliana (Cárdenas, 2011).

Las que forman parte de la red nacional tienen como objetivo ser la voz de la universidad y emiten programas "de carácter humanista, sin fines de lucro que armoniza la entretención, educación, información y cultura, contribuyendo así a la formación de audiencias críticas y más exigentes" (REUCH, 2015).

Por otra parte, existen casos independientes, no reconocidos por la red oficial, que sin embargo realizan una importante labor desde la universidad. Casos como el de la Radio Comunitaria Juan Gómez Millas, que desde 2004 transmite producciones realizadas por estudiantes y profesores de Periodismo del Instituto de la Comunicación e Imagen de la Universidad de Chile. El contenido está pensado para la comunidad vecina, pero también ofrecen una mirada crítica de los problemas sociales del país y participan activamente en producciones colectivas con otras radios comunitarias.

Se observa también en este panorama, arropados por la Internet, radios estudiantiles de instituciones como Universidad Diego Portales, que buscan la práctica radiofónica, pero también la presencia de la universidad en el mercado radiofónico online. 


\section{Ecuador: una red naciente}

El origen de la radio universitaria ecuatoriana se registra en la década de los cincuenta, en los centros de producción de las escuelas de Comunicación social y periodismo, donde los estudiantes tenían sus laboratorios de medios. "Luego, al darse cuenta del potencial humano y tecnológico con que disponían las universidades surge la idea de gestionar de mejor forma el laboratorio y es cuando se aventuran a emitir en potencia baja una señal para el campus universitario" ( Yaguana y Aguiló, 2014, p. 73).

Posterior a ello, se empezaron a pedir frecuencias que se vieron frenadas por el ritmo que conllevaba y los objetivos se perdieron en algunas universidades que se habían distinguido por ofrecer una radio equilibrada, con cobertura en las cafeterías y cercanías de la universidad.

Uno de los proyectos más grandes nace en 2006, como parte de una propuesta integral de medios de la Universidad Católica de Santiago de Guayaquil. Bajo el nombre de UCSG Radio-Televisión, es considerado el primer medio de comunicación universitario, integrado a nivel nacional, del que otros centros educativos han buscado inspiración (Bonoso, García y Pérez, 2011). Esta radio es realizada por estudiantes de los últimos ciclos de las carreras de arte y humanidades, y comunicación social; profesores y académicos tiene una cobertura regional, "con una audiencia aproximada de 35.000 personas, lo que la coloca en el puesto 11 de las emisoras de Guayaquil" (Reyes, 2014), algo inusual en las radios de este tipo.

Con la llegada de las nuevas tecnologías y nuevos ánimos en los centros universitarios, además de la ley que permite la figura de radios comunitarias, a partir de 2013, la radios universitarias de Ecuador que suman 9 por internet y dos en $A M$, deciden reunirse $y$ formar oficialmente la Red nacional, integrada por emisoras públicas y privadas.

Con el respaldo de expertos e intelectuales del medio como José Ignacio López Vigil, la radio se presenta a sí misma como enfocada a "contribuir al acercamiento, desarrollo y fortalecimiento de las relaciones académicas y comunicacionales entre todos los integrantes de la red" (RRUE, 2015).

La radio ecuatoriana tiene un rostro jovial y estudiantil, que tiene relevancia en su entorno. Ejemplos son Radio Universidad de Bolívar, "donde los estudiantes con buenos talentos han sido estrictamente escogidos y entrenados para mantenerse dentro de la 
radio" (Bonoso, García y Pérez, 2011); o Radios Cocoa y Radio Activa, consideradas como dinámicas y más "enriquecedora que la radio comercial” (Reyes, 2014).

También destaca la radio de la Facultad Latinoamericana de Ciencias Sociales (FLACSO), que tiene una frecuencia online realizada por un equipo de estudiantes internacionales de posgrado que ofrecen una programación crítica "para socializar procesos, comentar publicaciones, selecciones de música y archivos sonoros en diálogo con las temáticas tratadas (...) y contribuir a la formulación de agendas públicas participativas" ( FLACSO Radio, 2015).

\section{Otras radios en países de América Latina}

La experiencia de hacer radio desde las Universidades existe prácticamente en todos los países de América Latina, sin embargo no han destacado de manera numerosa, como otros.

De estas breves experiencias, destaca Costa Rica, donde existen algunas emisoras, como la de Radio Universidad nacida en 1949, que se distingue por ser una radio institucional adscrita a la Vicerrectoría de Acción Social, interesada en la difusión artística y educativa "que garantiza el respeto de la libre manifestación de ideas y opiniones y una programación creativa y de calidad" (Radio UCR, 2015). Aquí también existe un fuerte impulso por las emisoras y productoras estudiantiles a quienes se les ofrece la opción de "espacios para expresar y compartir sus ideas y opiniones, música, programas informativos, culturales y de análisis" (Araya, 2009).

Venezuela, de quien se refiere que cuenta con sus primeras emisoras universitarias en los años 90 (Paz, Romero y Díaz, 2005), para 2007 ya tenía diez con frecuencia en FM y un perfil en su mayoría "institucional" y donde es posible ubicar el modelo comercial, adjudicado a universidades privadas, "Aunque formalmente la radio universitaria no se catalogue como institucional debido a que existen también emisoras universitarias privadas, siempre serán institucionales por sus objetivos; aunque no necesariamente institucionalistas" ( Castro y Pineda, 2007).

En Cuba por ejemplo, se suman también numerosas radios - por Internet o como talleres universitarios, en su mayoría-, que apoyadas por la radio pública cubana, se reúnen bianualmente para celebrar el Festival Nacional de la Radio Universitaria, que lleva nueve emisiones. Allí se premian las producciones y se abre una convivencia cercana entre estudiantes que realizan esta labor sonora en toda la isla. La radio universitaria cubana se 
caracteriza por estar en manos de los estudiantes y por ofrecer contenidos relacionados a la vida universitaria. En su convocatoria de 2014 participaron 80 delegados con más de 140 trabajos (Radio Cubana, 2014).

Por su parte, Bolivia es distinguido por ser uno de los países con más emisoras de radio del continente después de Brasil y México. También existen emisoras universitarias de reciente creación como UCBradio de la Universidad Católica Boliviana y radio UB de la Universidad Unión Bolivariana, ambas privadas y por internet.

En Puerto Rico, donde solamente existen tres radios de tipo pública, dos de ellas son propiedad de universidades: La WRTU de la Universidad de Puerto Rico y la WEUC de la Católica de Puerto Rico. La primera se define como una cadena de emisoras educativas donde practican los estudiantes de la escuela de Comunicación Pública del Recinto de Río Piedras, con la misión de "servir a la comunidad mediante programación que eduque, informe y entretenga" (WRTU, 2015)y la segunda, como un vínculo entre los estudiantes y la sociedad a través de una programación de adulto contemporáneo y religioso.

Finalmente, se destaca la labor de la radio de San Carlos de Guatemala, la única emisora con perfil diferente en su país que abrió las puertas a la academia y las agrupaciones educativas y culturales (de León, 2007) y Radio Cáritas, de la Universidad Católica del Paraguay, que ha tenido una larga historia formativa, evangelizadora y a la vez con un departamento de publicidad que le permite generar ingresos.

\section{España: la apuesta tecnológica}

Por último, llegamos al caso de España, donde la radio universitaria, como se anotó al inicio, se desarrolló con mayor fuerza a penas a partir de 1995, con la facilidad que trajo hacer radio por Internet. Los talleres de las facultades de comunicación de sus universidades fueron el principal nicho, pero no el único para la expresión de la comunidad universitaria. Las iniciativas vinieron de asociaciones estudiantiles y posteriormente como salida de sus talleres de radio, por lo que la función universitaria primordial ha sido la formación profesional.

Muchos proyectos han desaparecido a lo largo de los últimos 20 años, por tratarse de iniciativas que se promovían desde el ámbito escolar y posteriormente por la crisis económica del país. Actualmente se contabilizan alrededor de 34 (Martin-Pena y 
Contreras-Pulido, 2014) aunque pertenecen a la ARU, red de emisoras del país, solo 23 radios de universidades públicas y privadas, la mayoría por internet.

Entre las características particulares que encontramos es que combinan funciones esenciales: la formación, a través de talleres escolarizados, con la difusión cultural y científica y el servicio público. A pesar de que se padece la falta de personal, de recursos económicos y de una ley que ampare su existencia, existe un gran espíritu de trabajo con los jóvenes (Vázquez, 2012). El uso de tecnología les ha dado un plus que les permite romper barreras y ampliar la experimentación en formatos como el podcast, la radio a la carta, las aplicaciones para teléfonos inteligentes, los servicios interactivos y las redes sociales(Marta-Lazo y Martin-Pena, 2014).

En cuanto al contenido que emiten, la mayoría presenta una parrilla con más de 20 espacios diferentes, de tipo mosaico, es decir con programas temáticos donde destaca los magazines de actualidad, con contenidos básicamente culturales y sociales; la música en diferentes géneros, sin faltar la actualidad universitaria y la divulgación de la ciencia y tecnología (Marta y Segura, 2012; Espino Narváez, 2014).

\section{Las redes}

Sin duda, la conformación de redes por países y recientemente por regiones, ha permitido que la radio universitaria tenga una imagen de fortaleza y de trascendencia en su ámbito. Juntas, con las condiciones que de cada experiencia, -SINPRIES en México; ARUNA en Argentina; REUCH en Chile; RRUC en Colombia; ARU en España y RRUE en Ecuador-, han logrado importantes avances y beneficios que de manera aislada no hubieran sido posibles.

La necesidad de internacionalización llevó a la conformación de la RRULAC en 2010, con el fin de fortalecer la radio de las instituciones universitarias dentro del contexto latinoamericano y su función social con los objetivos de propiciar alianzas internacionales; fomentar y facilitar el intercambio de producciones y experiencias y desarrollar proyectos cooperativos que potencien su impacto, entre otros (RRULAC, 2014).

En los últimos años estas redes se han visto fortalecidas con proyectos mundiales, como la celebración de la radio universitaria en sus dos versiones: La que incluye a los college radio de Estados Unidos, denominado World College Radio Day, que se celebra en los primeros días de octubre y a partir de 2013, el día de la radio universitaria latinoamericana, instituido 
el 5 de abril, conmemorando el aniversario de la radio más antigua. Otros esfuerzos están en proceso, dada la activación que también existe en otras redes como $A R U$ de España, RadUni de Italia y la francesa Radio Campus Francia, que buscar dialogar y contribuir en demostrar al mundo la importancia de la radio hecha desde las casas de estudio.

\section{Los retos}

Si bien la tecnología ha traído mayor visibilidad y presencia a la radio universitaria, su audiencia sigue siendo imprecisa y más diversificada, dada la oferta a la que tiene acceso todo ciudadano. Su labor a veces se desdibuja en ser entre un mero instrumento político, institucional o un laboratorio experimental, sin tomar en cuenta los intereses de la audiencia. Su programación parece a veces de espaldas al oyente, cuando la radio debe propiciar el desarrollo humano y "promover a través de la comunicación un nuevo sujeto sociocultural, una comunicación para el cambio social y para la construcción de la ciudadanía comunicativa plena". (Novelli y Hernando, 2011, p. 24).

Sobran razones para justificar la existencia de la radio universitaria, pues desde sus objetivos primarios se tiene claro que es para contribuir en la formación, a la democratización de las comunicaciones; para llevar contenidos no comerciales a provincias y regiones donde no hay emisoras públicas ni comunitarias; para dar voz a los sin voz; para llevar la educación a quien la necesita. No obstante, es vulnerable en ciertos puntos, como la limitación de recursos institucionales y el impedimento de comercializar espacios en algunos países.

Por otra parte, la radio universitaria, en muchos casos, aún debe tener en cuenta que es necesario cambiar de fondo para poder sobrevivir y destacar en este momento histórico. Para ello creemos que es necesario evitar lo siguiente:

a) La burocratización de sus trabajadores y por lo tanto de sus producciones.

b) La cerrazón de emitir contenidos elitistas, que no dan voz a su comunidad y sus interés y problemas.

c) La desvinculación con la tecnología y las dinámicas de las audiencias contemporáneas.

Es necesario, también, adoptar nuevos esquemas de trabajo y modos de producción que permitan la interacción con sus usuarios - antes denominados audiencia, por su estado pasivo-, y trabajar formatos híbridos que permitan su difusión por otros medios. 
En cuando a contenidos, como afirma Aguaded (2014), "se tiene el reto y la urgencia de aportar luz y desarrollo a la sociedad, la ciencia y el saber, como motores de progreso y transformación social", con un toque fresco, cercano a la gente y sin mayor pretensión que ser esa ventana que refleja lo que toda universidad es: conocimiento, diversidad y libertad de expresión.

\section{A manera de conclusión}

Como se puede observar, la radio universitaria en Iberoamérica está numéricamente bien representada, viva y aportando de manera significativa en la democratización de las comunicaciones, con su presencia en cada uno de los países de la región. En Argentina destacan las emisoras institucionalizadas con participación social y expresión política; en México las radios de múltiples perfiles que emiten contenidos culturales y académicos; en Colombia la convivencia de la radio universitaria de interés pública y la privada-comercial con alta presencia estudiantil; en Chile se identifican voces críticas desde las universidades nacionales y las radios comunitarias y en Ecuador, la radio por Internet se abre paso para que la comunidad estudiantil tome el micrófono.

Esto, aunado a la fortaleza que han tomado al organizarse en redes que buscan diálogo con otros grupos, y los demás ejemplos mencionados, nos demuestra la trascendencia que ha tomado esta radio que es considera vital y necesaria en muchas regiones donde no hay alternativas, frente al apabullante sistema comercial de medios; sin embargo aún tiene grandes retos que muy pocas radios han logrado superar en los últimos años.

Como se mencionó, son retos económicos, tecnológicos y de actitud de cambio para las formas de producción, pero sobre todo de contenido. La radio, en su mayoría, es vocera de la institución y de la cultura en todas sus expresiones; y cada vez más de la juvenil, pero se observa que es menos comprometida con su entorno social que otras radios no comerciales y eso, puede afectar su futuro pues, como afirma Montells (2010), "Si algo debiera preguntarse una radio, es cuál es la tarea de su época. Además de medios de comunicación, las radios son actores sociales que construyen sentido y movimientos. Espacios para recuperar la curiosidad, el asombro y la justicia". 


\section{LA RADIO UNIVERSITARIA EN IBEROAMÉRICA. Marina Vázquez Guerrero}

Referencias Aguaded, J.I. (2014) Prólogo. EnMartín Pena, D. y Ortiz Sobrino, M.A. (comps.) Las radios Universitarias en América y Europa. Madrid: Fragua.

ANUIES, y UNESCO. (2007). Radiodifusoras y Productoras de las Instituciones de Educación Superior en México. Los Medios de Comunicación Universitarios en México 2005[CD]. ANUIES Y UNESCO.

Araya(2009) Radio estudiantil: programas, audiencias y desafíos. Reflexiones 88 (2) 37-44.

ARUNA (2014) La primera radio universitaria de Iberoamérica cumple 90 años. http://www.aruna.org. ar/noticias/interior.php?id=142

Berlín, I. (2000), El derecho a decir: radios universitarias y educativas en México. Revista Latina de comunicación social, 27. Recuperado de: http://ull.es/publicaciones/latina/aa2000tma/125/irving. html

Bonoso, V:; García, R. y Pérez, H. (2011) Diseño y producción del programa de radio para la facultad de filosofía, letras y ciencias de la educación 2011. (Trabajo final de Licenciatura en Comunicación Social. Universidad Católica de Santiago de Guayaquil). Recuperado de:http://repositorio.ucsg.edu.ec/ bitstream/123456789/406/1/T-UCSG-PRE-FIL-CCS-7.pdf

Capote, L. (2010). Entrevista personal al director de Radio Campus de la Universidad de la Laguna.

Cárdenas J.P.(2011)Las cifras de la radio que piensa. Recuperado de: http://izkias.blogspot.com/2011/01/ las-cifras-de-la-radio-que-piensa.html

Casajús, L. (2011) Radio Universitaria en América Latina: Escenario y perspectivas. En Aguaded, J.I. y Contreras-Pulido (coord.) La radio universitaria como servicio público para una ciudadanía democrática. La Coruña: Netbiblo.

Castro, E. y Pineda, A. (2007)La política comunicacional de las universidades venezolanas y su impacto sobre la competitividad de sus emisoras de radio. Revista de Ciencias Sociales v.13 n.3 Maracaibo.

Chaparro,M.(1998). Radio pública local. Sevilla:Fragua.

Dahma,D.(2014)El trabajo en red en Latinoamérica: LaRRULAC. En Martín Pena, D. y Ortiz Sobrino, M.A. (compiladores) Las radios Universitarias en América y Europa. Madrid,Fragua.

Dido,J.C.(2008)Radiosuniversitarias: realidad y perspectivas. 10 congreso REDCOM.Conectados, Hipersegmentados y Desinformados en la Era de la Globalización. Salta, Argentina.

De León, V.H. (2007) “La radio en Guatemala” en Merayo (Ed.).(2007). Radio en Iberoamérica, evolución, diagnóstico y prospectiva. España: Comunicación Social ediciones y publicaciones.

Espino Narváez, C. (2014). Contenido, estructura y función en la programación de las radios universitarias españolas. EDMETIC, Revista de EducaciónMediática y TIC, 3(1), 26-43.

Faus,Á.(1973). Laradio: Introducción al estudio de un medio desconocido.Madrid: Guadiana de Publicaciones.

Fidalgo,D.(2009).Las radios universitarias de España. Transformación al mundo digital. Telos80. Recuperado de :http://sociedadinformacion.fundacion.telefonica.com/telos/articulodocumento. asp@idarticulo=2yrev=80.htm 
FLACSO radio ( 2015) Emisora universitaria de la Facultad Latinoamericana de Ciencias Sociales en Ecuador. http://www.flacsoradio.ec/

Gaviria,G.(2008)LaradiouniversitariaenColombia. Revistalaveriana2008.

Jiménez,C.yMuñoz,J.(2008)Estructura de los medios de comunicación en Chile. Razón y Palabra, vol.13, núm.60, enero-febrero.

LT10(2015)Página web oficial de la radio de laUniversidad Nacional del Litoral, Argentina.http://www. Ir11.com.ar/

Magaña López, S. (2003)El modelo de radio universitaria en Estados Unidos de Norteamérica: el caso de KTCU. (Tesis de Licenciatura. Ciencias de la Comunicación. Universidad de las Américas, Puebla, México). Recuperado de: http://catarina.udlap.mx/u_dl_a/tales/documentos/lco/magana_I_sm/ portada.html

Marta - Lazo, C. y Martín - Pena, D. (2014) Antecedentes, estado de la cuestión y prospectiva de las Radios Universitarias. Edmetic,3(1).

Marta, C. y Segura, A. (2012). Emisoras universitarias españolas en la Web 3.0:programación y contenidos En Espino, C. y Martín, D. (coords.). Las radios universitarias, más allá de la radio. Las TIC comorecursos de interacción radiofónica. Barcelona: UOC.

Martí,J.M.(1995).Nuevos formatos de radio en USA: Jornada profesional: Texto, ponencias.Barcelona: Fira de Barcelona.

Merayo(Ed.).(2007).Radio en Iberoamérica, evolución, diagnóstico y prospectiva. España:Comunicación Social ediciones y publicaciones.

Milito, C., y Casajús, L. (2011). Las radios universitarias argentinas en internet: relevamiento, desarrollos, modelos y enfoques. Question, 1(30). Recuperado dehttp://perio.unlp.edu.ar/ojs/index.php/question/ article/view/1150/1057

Miller,J.(2014)AChronologyofAMRadioBroadcasting1900-1960.Blog. Recuperado de: http://jeff560. tripod.com/chrono1.html

Montells, G. (2014) Toda esa radio del mundo.Trampas de la Comunicación 83. Universidad Nacional de La Plata.

Morales,P. (2008). Audiencias de radios universitarias y alternativas en hegemonías comunicacionales. En B.Ammann, y E.DaForta (Eds.), Rutas alternativas de la comunicación.Procesos de significación social e ideológica y poder.(pp.95). BuenosAires, Argentina: Ferreyra editor.

Novelli,C.yHernando,A.(2011)Lasradiosuniversitarias. Trayectoriahistóricaypanoramamundial. EnAguaded,J.I.yContreras-Pulido(coord.)LaCoruña.Netbiblo.

Paz,A.Romero,S.yDíaz,B.(2005)Discreción de los factores de rentabilidad de los medios universitarios. Formación gerencial Ano. 4, no.1

PrietoCastillo,D. (1996) Las emisoras Universitarias frente a las transformaciones de fin de siglo.IFestival Centroamericano de la radio. Costa Rica. Recuperado de: http://www.monografias.com/trabajos912/ las-emisoras-universitarias/las-emisoras-universitarias.shtml 
RadioCubana(2014)"Apropósito de IIX Festival de la RadioUniversitaria". http://www.radiocubana.icrt. cu/index.php?option=com_content\&view=article\&id=4381\%3Aa-proposito-del-ix-festival-de-laradio-universitaria\&catid $=9 \&$ Itemid $=127(27 / 05 / 2014)$.

Radio UCR (2015)Página web oficial de la Radio Universidad de Costa Rica.http://radios.ucr.ac.cr/ radio-universidad/la-radio.html(03/02/2015).

REUCH(2015)Página web oficial de la Red de Radioemisoras Universitarias de Chilehttp://www.reuch. $\mathrm{Cl} /(02 / 04 / 2014)$

Reyes, J. (2014) Las radios universitarias en Ecuador. Wikispaces. https://historia-radio-tv.wikispaces. com/Las+radios+universitarias+de+Ecuador

Rotman,A.(2014) Las radios universitarias, Argentina frente a un nuevo paradigma de la comunicación. En Martín Pena ,D. y Ortiz Sobrino, M.A.(comps.) Las radios Universitarias en América y Europa. Madrid: Fragua.

RRUE(2015)Página web oficial de la red de Radios Universitarias del Ecuador http://www.rrue. org/?page_id=78(06/02/2015).

RRULAC(2014)Página web oficial de la administración 2012-2014 https://sites.google.com/a/rrulac. org/rrulac/principios-organizativos(04/02/2015).

SINPRIES (2015) Página web oficial del Sistema Nacional de Productoras y Emisoras de las Instituciones de Educación Superior de México. http://sinpries.red.anuies.mx/ (03/01/2015).

Slotten,R.H. (2006). Universities, public service radio and the americans y stem' of commercial broadcasting, 1921-40. Media History,12,253-272.

UNED(2015)Páginaweboficialde la Universidad Nacional de Educación a Distancia http://portal.uned. es/portal/page?_pageid=93,499271\&_dad=portal\&_schema=PORTAL

Vázquez Guerrero, M. (2012). La radio universitaria en México y España. Estudio de la participación y formación de los jóvenes. (Tesis doctoral. Universidad Pompeu Fabra). Recuperado de: http://www.tdx. cat/bitstream/handle/10803/84113/tmvg.pdf;jsessionid=E678DAB59897B0CF71BB2F4492356D2A. $\operatorname{td} x 1$ ? sequence $=1$

Vázquez Guerrero,M. y Chamizo, F. (2014) LaRadio Universitaria en México: diversidad y contraste. En Martín Pena, D. y Ortiz Sobrino, M.A. (comps.) Las radios Universitarias en América y Europa. Madrid: Fragua.

Yaguana,H. y Aguiló, J.M. (2014) La radio universitaria ecuatoriana, un reto para un nuevo tiempo. En Martín Pena, D. y Ortiz Sobrino, M.A. (comps.) Las radios Universitarias en América y Europa. Madrid, Fragua.

Zambrano,W. (2012) Radiografía de las emisoras universitarias colombianas. Folios 28. Facultad de Comunicaciones. Universidad de Antioquia pp.115-138

WPR (2014) Página web oficial de WPR http://www.wpr.org/about-wpr / (13/12/2014).

WRTU (2015) Página web oficial de Cadena Radio Universidad de Puerto Rico http://www.wrtu.pr 